\title{
Besteht ein Bezug zu Alzheimer und Parkinson?
}

Mit ihren 3 Postulaten prägten Robert Koch und Jakob Henle das Bild von Infektionskrankheiten:

- Eine Infektionskrankheit ist Ausdruck der Tätigkeit eines Erregers.

- Dieser lässt sich aus einem Patienten isolieren und in geeigneten Nährmedien vermehren.

- Bei seiner Rückübertragung in ein empfindliches Individuum erzeugt er erneut das ihm entsprechende Krankheitsbild.

Die klassischen Krankheitserreger wie Bakterien und Pilze erfüllten diese Kriterien uneingeschränkt. Dagegen schienen sich Viren der zweiten Regel zunächst zu widersetzen - bis es gelang, Wirbeltierzellen in Kultur zu nehmen (Abb. 1). Erst mithilfe solcher Zellkulturen glückte es in den 60er-Jahren des letzten Jahrhunderts, auch Viren außerhalb ihrer Wirtsorganismen zu vermehren.

\section{Neue Krankheitserreger}

Zunächst dachte man, dass nun alle großen Kategorien von Krankheitserregern entdeckt worden seien und erwartete hier keine weiteren bedeutenden Entde- ckungen mehr. Schon bald zeigte sich jedoch, dass es weitere übertragbare Erkrankungen gab, die man nicht den klassischen Virusinfektionen zuordnen konnte. Diesen gab man zunächst den $\mathrm{Na}$ men „Slow Virus Diseases“. Aber bald schon war es erforderlich, sie in „Slow Infections“ umzubenennen, weil vieles im Ablauf der Erkrankungen und in den Eigenschaften der Erreger beträchtlich von dem, was klassische Virusinfektionen, zeigten, abwich.

Daniel Carleton Gajdusek war derjenige, der dies als einer der Ersten erkannte und dem im Jahre 1976 hierfür der Nobelpreis für Medizin verliehen wurde. Er war es auch, der nachwies, dass Erkrankungen wie die Creutzfeldt-Jakob-Erkrankung, Kuru, Scrapie und einige weitere Krankheiten beim Menschen und Nutztieren nicht von klassischen Erregern verursacht werden konnten, sondern von einer völlig neuartigen und offensichtlich noch unbekannten Kategorie von Erregern. Danach dauerte es weitere 21 Jahre, bis man mit Stanley Prusiner denjenigen mit einem zweiten Nobelpreis ehrte, der die neuen Erreger als Prionen, d.h. als infektiöse Eiweiße mit einer autokatalytischen Aktivität, beschrieb.

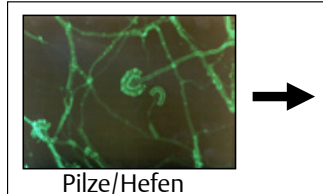

Pilze/Hefen

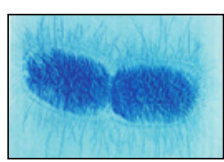

Bakterien

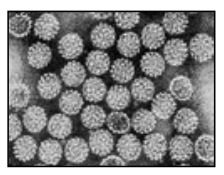

Viren
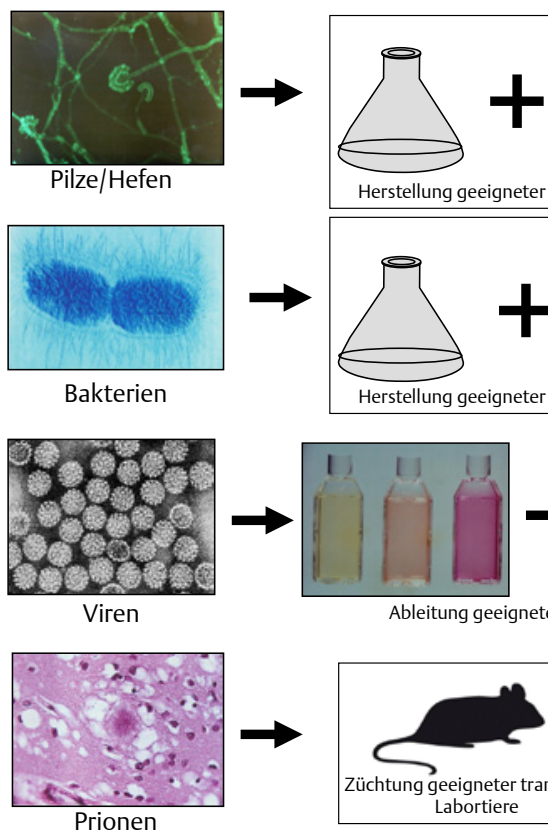

Herstellung geeigneter Nährlösungen/-böden
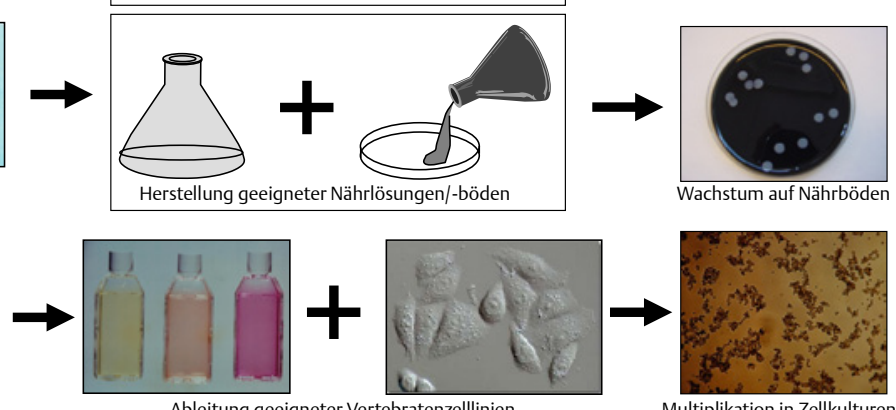

Ableitung geeigneter Vertebratenzelllinien Multiplikation in Zellkulturen
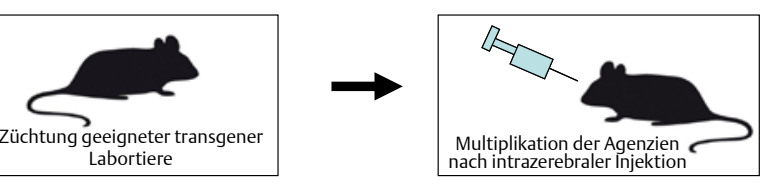

Abb. 1 Darstellung der unterschiedlichen Arbeitsweisen bei der Anzucht der jeweiligen Erreger in Bakteriologie/Mykologie, Virologie und Prionenforschung.
Prionen - die ungewöhnlichen Erreger Prionenerreger stellen in vielerlei Hinsicht das durch klassische Erreger skizzierte Bild der Infektionskrankeiten auf den Kopf. Dies beginnt schon bei der Trennung der Begriffe „Übertragbarkeit“ und „Infektiösität“. Während man eine diesbezügliche Differenzierung bei Pilzen, Bakterien und Viren als reine Semantik abtun kann - ein übertragbarer Erreger ist hier auch als infektiös einzustufen -, ist eine solche Gleichstellung bei Prionen nicht mehr so einfach möglich. Gelingt zum Beispiel eine Übertragung eines Prions im Tierexperiment, so kann man über die Infektiösität des Spenders unter natürlichen Bedingungen immer noch keine gesicherte Aussage machen. Eine weitere Besonderheit von Prionenerkrankungen ist das Vorkommen hereditärer (d.h. „angeborener“) Formen. Im klassischen Bild einer Infektionskrankheit führen Erreger und Wirt voneinander trennbare Existenzen: So gibt es den potenziellen Wirt, der solange nichts zu befürchten hat, solange er nicht Kontakt mit dem Erreger bekommt. Und es gibt den Erreger, dessen Bestrebung es ist, einen geeigneten Wirt zu finden, um sich mit dessen Hilfe zu vermehren. Bei Prionen ist selbst diese Verkettung von Wirt und Erreger nicht mehr generell gegeben. So kann ein Wirt, welcher über entsprechend mutierte Gene verfügt, im Verlauf seines Lebens spontan Prionenerreger generieren, die ihn dann erkranken lassen und am Ende töten. Mit geeigneten Patientenmaterialien kann der so entstandene Erreger zumindest unter Laborbedingungen auf weitere empfängliche Individuen übertragen werden. Ein zuvor vollkommen gesunder Wirt kann also spontan zur „Geburtsstätte“ eines übertragbaren Erregers werden.

\section{Eigenschaften von Prionen}

Prionen gibt es in mindestens 2 Modifikationen: die gesunde und die krankhafte Form. Bei beiden Formen handelt es sich um Eiweißmoleküle. Die gesunde Form entsteht offensichtlich bei allen Säugetieren und hier vor allem in Hirnzellen. Diese Prionen werden durch Proteinbiosynthese im Plasma der Zellen gebildet und erfüllen anschließend in der Oberfläche der gleichen Zellen eine noch weitgehend unbekannte biologische Funktion.

Als Eiweiße werden Prionen aus Aminosäuren zusammengesetzt und erhalten bei ihrer Entstehung neben der durch die zel- 
luläre DNS festgelegten Aminosäuresequenz zusätzlich auch noch eine ganz spezifische Raumstruktur. Der Bauplan, nach dem „gesunde“ Prionen entstehen, ist also in der DNS im Zellkern hinterlegt. Die Existenz von Prionen ist somit zunächst kein Zeichen für ein krankhaftes Geschehen, sondern etwas völlig Selbstverständliches. Entspricht nun die Aminosäuresequenz eines Prionproteins zwar dem Bauplan im Zellkern, weicht jedoch die Raumstruktur von der natürlicherweise vorgesehenen Struktur ab, so kann das entsprechend veränderte Prionprotein die folgenden fatalen Eigenschaften erhalten:

- Es nimmt seine natürliche Funktion nicht mehr wahr.

- Es erhält eine katalytische Aktivität und vermag in engem Kontakt mit gesunden Prionproteinen diesen seine krankhafte Form aufzuzwingen.

- Es kann in den Zellen nicht mehr abgebaut werden.

- Es bildet Aggregate, die sich als intrazelluläre Einschlusskörperchen in den betroffenen Zellen anreichern und diese schließlich zerstören.

- Es gelangt in weitere Zellen und setzt den Vorgang dort fort.

\section{Prionenforschung}

Um krankhafte menschliche Prionen nachzuweisen oder diese in größeren Mengen anzuzüchten, ist man v.a. auf Versuchstiere angewiesen, obgleich es inzwischen auch gute In-vitro-Nachweise gibt. Üblicherweise gewinnt man Ausgangsmaterial aus dem Hirn eines erkrankten oder verstorbenen Patienten und überträgt etwas Material davon auf geeignete Versuchstiere. Am besten gelingt dies durch intrazerebrale Verimp-

Tab. 1 Gesicherte Prionenerkrankungen.

\begin{tabular}{|l|l|}
\hline $\begin{array}{l}\text { Krankheit } \\
\text { Kuru }\end{array}$ & $\begin{array}{l}\text { Spezies } \\
\text { Mensch }\end{array}$ \\
\hline Creutzfeld-Jakob-Erkrankung & Mensch \\
\hline Gerstmann-Sträussler-Syndrom & Mensch \\
\hline bovine spongiforme Enzephalopathie & Rind \\
\hline Scrapie & Schaf \\
\hline spongiöse Nerzenzephalopathie & Nerz \\
\hline Chronic Waisting Disease & $\begin{array}{l}\text { Schwarzwedelhirsch } \\
\text { Wapiti-Hirsch }\end{array}$ \\
\hline spongiforme Enzephalopathien & Nyala \\
& Elen-Antilope \\
& Oryx-Antilope \\
& Kudu \\
\hline
\end{tabular}

fung in das Hirn von Hamstern oder Mäusen.

$\mathrm{Zu}$ einem Durchbruch in der Prionenforschung kam es allerdings erst, als es möglich wurde, natürliche Prionengene des Menschen in das Erbgut von Versuchstieren einzuschleusen. Bei diesen sogenannten transgenen Tieren gelingt die Übertragung besonders gut und es kommt schon nach kurzer Zeit zum Auftreten der typischen Krankheitssymptome. Eine Vorstellung von der Arbeitsweise in der Prionenforschung gibt Abbildung 1.

Schon seit Langem wurde spekuliert, dass auch anderen Erkrankungen, bei denen sich ähnliche intrazelluläre Einschlusskörper in Hirnzellen beobachten lassen, ein vergleichbarer Mechanismus zugrunde liegt. Dies wurde vor allem für einige, keineswegs seltene Altersdemenzen diskutiert. Tatsächlich spricht inzwischen eine Fülle übereinstimmender experimenteller Daten dafür, dass eine keimabhängige Proteinpolymerisation nicht nur bei der CJD (Creutzfeldt Jakob Disease) und anderen gesicherten Prionenerkrankungen bei Menschen und Nutztieren stattfindet, sondern auch der Alzheimer- oder der Parkinsonschen Erkrankung und darüber hinaus noch weiteren Hirnerkrankungen zugrunde liegt (Tab. 1). Mit der Züchtung entsprechender transgener Versuchstiere, denen die Gene der jeweiligen Erreger in ihr Erbgut eingefügt wurden, lassen sich jedenfalls auch entsprechende Krankheitsbilder nach Übertragung von Hirnmaterial induzieren.

In jüngerer Vergangenheit wurde berichtet, dass man sogar mit dem Blut von Alzheimerpatienten Krankheitseffekte bei entsprechend manipulierten transgenen Mäusen induzieren könne. Dieser Befund war Anlass für eine Stellungnahme des Arbeitskreises Blut im Bundesgesundheitsblatt [1]. In dieser Stellungnahme wird ausgeführt, dass die Befunde jedoch einer Bestätigung durch andere Arbeitsgruppen bedürften, bevor aus ihnen Konsequenzen für die tägliche Arbeitsweise im medizinischen Bereich gezogen werden könnten.

In der Tat wären diese erheblich, falls sich bewahrheiten sollte, dass Blut ein Übertragungsrisiko bergen würde. Für die klassischen Prionenerreger wie demjenigen der CJD gilt nämlich erst die Exposition gegenüber heißem
Krankheiten, verursacht durch keimabhängige Proteinpolymerisation

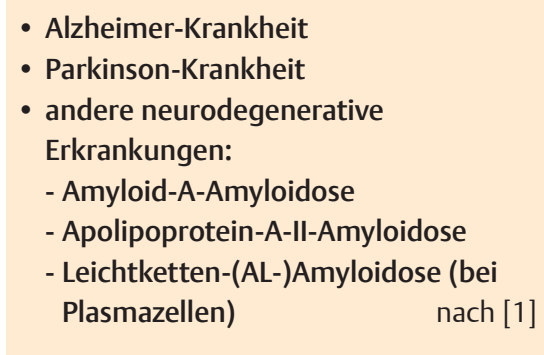

gespanntem Wasserdampf (Autoklavierung) bei $134^{\circ} \mathrm{C} / 5 \mathrm{~min}$ und einer zuvor erfolgten Behandlung mit einem hoch alkalischen Reinigungsverfahren als akzeptables Inaktivierungsverfahren. Es waren aber Prionenerreger, die noch vor gar nicht langer Zeit Anlass gaben, die zuvor angewendeten $121-{ }^{\circ} \mathrm{C}$-Dampf-Sterilisations-Verfahren auf $134-^{\circ} \mathrm{C}-$ Standardverfahren $\mathrm{zu}$ ändern.

Auch die auf $134{ }^{\circ} \mathrm{C}$ verschärften Autoklavierungsverfahren dürfen nur prophylaktisch angewendet werden. Werden nämlich Patienten mit einer gesicherten Prionenerkrankung medizinisch versorgt, so muss jegliches Material und Instrumentarium, welches bei der Behandlung mit dem Patienten in Kontakt gekommen ist, durch Verbrennung vernichtet werden. Hiervon sind lediglich Gastroskope ausgenommen, die mithilfe spezieller Desinfektionsmittel (Guanidiniumisothiocyanat) aufbereitet werden und die der Behandlung von CJD-Patienten vorbehalten bleiben (Ansprechpartner für Leihendoskope: Universität Göttingen, Abteilung Neuropathologie).

\section{Fazit}

Prionen sind sicherlich die interessanteste Gruppe von Krankheitserregern überhaupt. Manches spricht dafür, dass ihre Erforschung nicht nur das Bild einiger heute noch als rätselhaft eingestufter Krankheiten verändern wird. Hier steckt auch ein Potenzial, das unser Wissen in manch anderen wissenschaftlichen Disziplinen revolutionieren könnte.

PD Dr. Dr. Friedrich von Rheinbaben, Schwerin; Dr. Sebastian Werner, Bochum

\footnotetext{
Literatur

1 Mitteilungen des Arbeitskreises Blut des Bundesministeriums für Gesundheit. Sicherheit von Blut und Blutprodukten angesichts aktueller Berichte über die Übertragbarkeit der Alzheimer-Krankheit im Tierexperiment. Bundesgesundheitsbl 2013; 56: 584
} 